\title{
Effect of Turbulence Modeling on Hovering Rotor Flows
}

\author{
Seokkwan Yoon ${ }^{1}$, Neal M. Chaderiian ${ }^{2}$, Thomas H. Pulliam ${ }^{2}$, and Terry L. Holst ${ }^{3}$ \\ NASA Ames Research Center, Moffett Field, California 94035
}

\begin{abstract}
The effect of turbulence models in the off-body grids on the accuracy of solutions for rotor flows in hover has been investigated. Results from the Reynolds-Averaged Navier-Stokes and Laminar Off-Body models are compared. Advection of turbulent eddy viscosity has been studied to find the mechanism leading to inaccurate solutions. A coaxial rotor result is also included.
\end{abstract}

\section{Introduction}

Accurate prediction of rotorcraft performance in hover continues to be challenging for Computational Fluid Dynamics (CFD). Flows are inherently complex because a rotor blade can encounter its own tip vortex and the tip vortices of other blades. The distance from the rotor to the far-field boundary has a significant effect on the figure of merit (FM) prediction. The FM is a measure of rotor hovering efficiency, defined as the ratio of ideal power required to hover to the actual power required to hover. The asymptotic FM is reached when the far-field boundary is located about fifteen to twenty rotor radii away from the rotor. ${ }^{1}$ Also, it often requires twenty to thirty revolutions for a running mean of the FM to reach a quasi steady state even in free air. ${ }^{1}$ Hover simulations within a wind tunnel test section are more challenging than free-air cases because of close proximity of facility surfaces. The low-speed induced flows can require hundreds of revolutions to settle out initial startup transients. ${ }^{2}$

Direct Numerical Simulation (DNS) solves the time-dependent Navier-Stokes equations by resolving the full range of spatial and temporal scales of turbulence. Since DNS requires a large number of grid points to resolve the Kolmogorov dissipative scales, simulating high Reynolds number flow is not feasible even with today's most powerful supercomputers. Large Eddy Simulation (LES) requires less computational resources than DNS by modeling the smallest turbulent length scales using a sub-grid model while still grid-resolving the larger eddies. However, the grid requirements for high Reynolds number LES calculations are still impractical. Since the high cost of computation for pure LES comes from the near-wall region, hybrid models like Detached Eddy Simulation ${ }^{3}$ (DES) have been developed. The DES approach alleviates the difficulty of resolving the smallest scales by using a Reynolds-Averaged Navier-Stokes (RANS) turbulence model in the boundary layer and LES to grid-resolve the largest scales away from the wall. The DES turbulence model behaves like Smagorinsky's LES sub-grid model in the region outside the boundary layer. Rotorcraft simulations using the DES model have been successfully performed to capture the FM within experimental error. ${ }^{4}$ An alternative approach is to turn off all source terms in the off-body grid wake region. The Laminar Off-Body (LOB) model $^{5}$ assumes that the turbulent eddy viscosity that is generated elsewhere, e.g. the rotor blades, can still convect, but not be produced, dissipate, or diffuse. Both DES and LOB models produce accurate solutions and their FM results are comparable. ${ }^{1}$

The RANS equations require a closure by modeling the Reynolds stress. The one-equation Spalart-Allmaras ${ }^{6}$ (SA) RANS model is one of the models commonly used to compute the turbulent eddy viscosity (TEV) using the Boussinesq approximation. It has been known that the SA-RANS model produces mostly inaccurate solutions for flows with large separation regions by failing to resolve the dynamics of the dominant larger turbulent eddies. A recent study ${ }^{4}$ shows that while the RANS model failed to predict the FM accurately for all collectives, the discrepancy between the RANS results and the experimental data was largest when there was significant bladevortex interaction (BVI), i.e. when a vortex passes close to the blade and influences the turbulent boundary layer.

The OVERFLOW ${ }^{7-9}$ CFD code overcomes geometrical complexity by using overset grids. The use of near-body (NB) and off-body (OB) overset grids also allows for the use of different turbulence model options in different grid zones. In the present work, the solutions from RANS and LOB models for the OB grids are compared. The SARANS model is used in the NB grids in all cases. Of particular interest is the mechanism by which the RANS model under-predicts the FM.

\footnotetext{
${ }^{1}$ NASA Advanced Supercomputing Division

${ }^{2}$ NASA Advanced Supercomputing Division, AIAA Associate Fellow

${ }^{3}$ NASA Advanced Supercomputing Division (Retired), AIAA Fellow
} 


\section{Numerical Approach}

OVERFLOW solves the RANS equations on structured overset grids. The current time-accurate approach consists of an inertial coordinate system where NB curvilinear O-grids (rotor blades and hub) rotate through a fixed OB Cartesian grid system. The medium-sized XV-15 isolated rotor grid system used in this study is shown in Fig. 1. A uniformly spaced OB Cartesian grid surrounds the rotor blades and hub and resolves the rotor wake region of interest. Coarser "brick-grids" efficiently expand the grid system to the far field, where each successive brick grid is twice as coarse as its previous neighbor. The XV-15 isolated rotor consists of three highly twisted rotor blades and a simplified hub. Each rotor blade consists of three O-grids, one for the main rotor blade and two "cap-grids" for the inboard and outboard tips. The grid spacing normal to solid surfaces maintains $\mathrm{y}^{+}<1$. The numbers of grids and grid points used in this study are summarized in Table 1. Note that the resolved wake region has uniform grid spacing of $10 \% \mathrm{c}_{\text {tip }}$ (rotor blade tip chord length). Care is taken to make sure the NB grid spacing is similar to the OB grid spacing where the grids overlap.

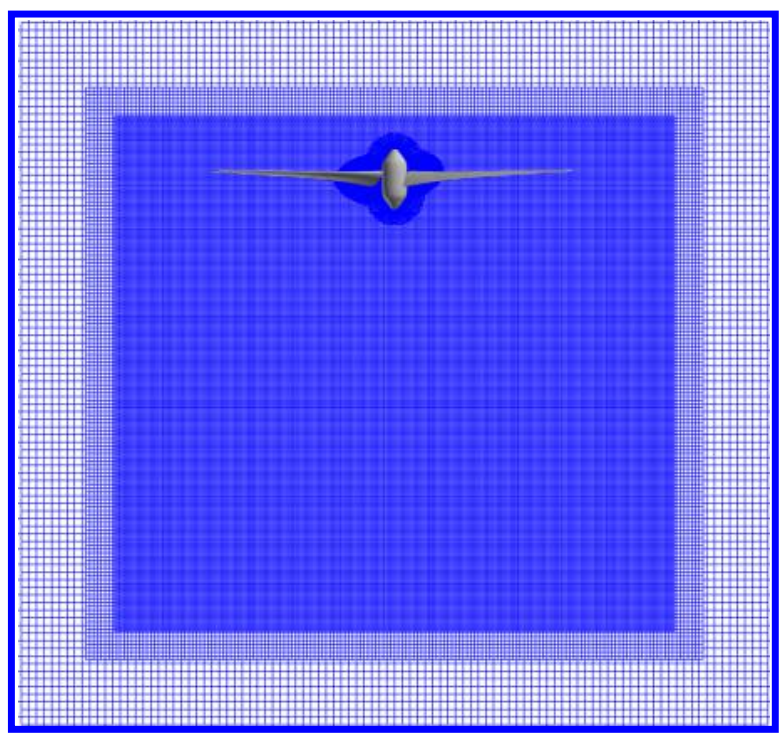

a) Fixed OB Cartesian grids

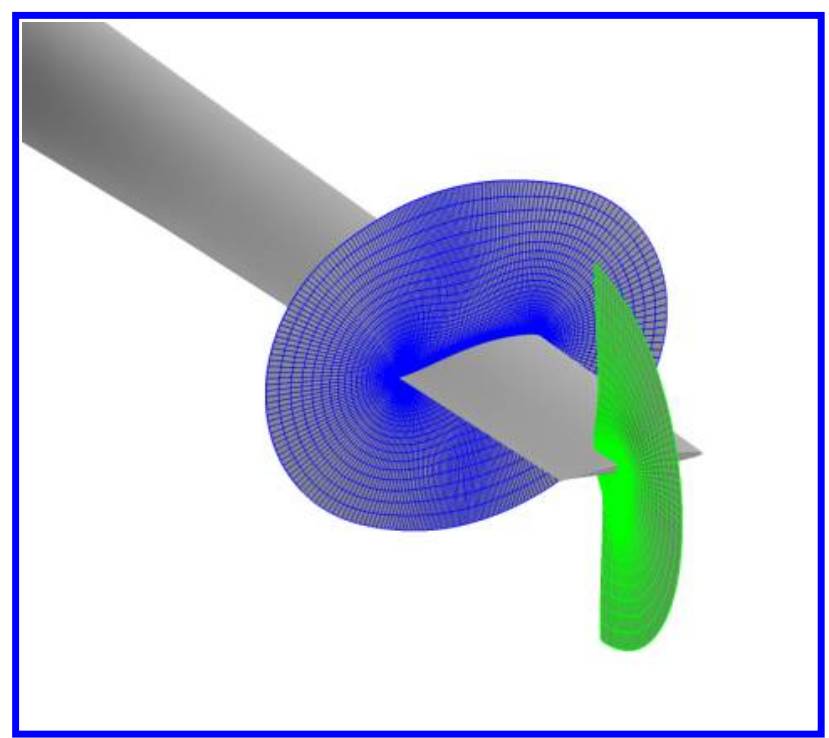

b) Rotating NB curvilinear rotor-blade grids

Figure 1. Overflow overset grid system for an isolated XV-15 rotor and simplified hub in hover

\begin{tabular}{|c|c|c|c|c|}
\hline Number of Grids & $\begin{array}{c}\text { NB Grid Points } \\
\text { (Million) }\end{array}$ & $\begin{array}{c}\text { OB Grid Points } \\
\text { (Million) }\end{array}$ & $\begin{array}{c}\text { Total } \\
\text { (Million) }\end{array}$ & $\begin{array}{c}\text { OB Resolution } \\
\text { (Percent } \mathbf{c}_{\text {tip }} \text { ) }\end{array}$ \\
\hline 62 & 18.6 & 28.6 & 47.2 & $10 \%$ \\
\hline
\end{tabular}

Table 1. Overflow overset grid system for the XV-15 isolated rotor and simplified hub.

OVERFLOW solves the Navier-Stokes equations using finite differences with a variety of numerical algorithms and turbulence models. ${ }^{7} \mathrm{Up}$ to $6^{\text {th }}$-order spatial accuracy for inviscid fluxes, up to $7^{\text {th }}$-order artificial dissipation, and $2^{\text {nd }}$-order time accuracy are available. ${ }^{8}$ In this study, the Pulliam-Chaussee ${ }^{10}$ diagonal central difference algorithm is used with the $5^{\text {th }}$-order spatial differencing option. ${ }^{8}$ Dual time-stepping is used to advance the simulation in time with $2^{\text {nd }}$-order time accuracy. This numerical approach and time step was previously validated for similar isolated rotor flows. ${ }^{4}$ In order to reduce the computational time required for a converged solution, the first 1,440 steps employ a time step of 2.5 deg, i.e., 10 rotor revolutions. The time step is then reduced to $0.25 \mathrm{deg}$, when 1,440 steps now correspond to one rotor revolution. The physical time step corresponds to 0.25 degrees rotor rotation, together with 30 dual-time sub-iterations. This typically provides for 2.5 orders of magnitude drop in sub-iteration residual. 


\section{Turbulence Modeling}

The SA-RANS model, implemented for the present study, uses the Boussinesq approximation to relate the Reynolds stresses to a kinematic turbulent eddy viscosity and the mean strain-rate tensor. The turbulent eddy viscosity is given by the expression

$$
v_{t}=\tilde{v} f_{v 1}
$$

The turbulence transport equation for the turbulence variable $\tilde{v}$, is given by

$$
\frac{D \tilde{v}}{D t}=P(\tilde{v})-D_{\text {diss }}(\tilde{v})+D_{\text {diff }}(\tilde{v})
$$

where $D / D t$ is the material time derivative. The right-hand side consists of turbulence production, dissipation and diffusion source terms. The production is given by

$$
P(\tilde{v})=C_{b 1} \tilde{v}\left(\Omega+\frac{\tilde{v}}{\kappa^{2} d^{2}} f_{v 2}\right)
$$

the dissipation by

$$
D_{\text {diss }}(\tilde{\boldsymbol{v}})=C_{w 1} f_{w}\left(\frac{\tilde{\boldsymbol{v}}}{d}\right)^{2}
$$

and the diffusion by

$$
D_{\text {diff }}(\tilde{v})=\frac{1}{\sigma}\left[\nabla \cdot((v+\tilde{v}) \nabla \tilde{v})+C_{b 2}(\nabla \tilde{v})^{2}\right]
$$

The constants $\mathrm{C}_{b 1}, \mathrm{C}_{b 2}, \mathrm{C}_{w 1}, \kappa$, and functions $f_{v 1}, f_{v 2}, f_{w}$, are described by Spalart and Allmaras. ${ }^{6}$ The damping function, $f_{v 1}$, reduces $v_{t}$ near a solid wall. Note that the OVERFLOW code uses the magnitude of vorticity, $\Omega$, in place of the strain-rate, since these two are approximately equal in a boundary layer and the vorticity is readily available in the OVERFLOW code. The turbulence length scale, $d$, is defined as the distance from a field point to the nearest wall. The accuracy of the SA-RANS model depends strongly on the source terms in Eq. (2), which were primarily developed for attached boundary-layer flows along flat plates, wings, fuselages, etc.

Chaderjian and Buning ${ }^{4}$ showed that the turbulence length scale, $d$, plays a key role in accurately determining the rotor FM. A problem occurs deep within the rotor wake, where $d$ may be several rotor radii in length. In this case, $d$ no longer represents an estimate of the largest turbulent eddy in the local flow, but rather, a very large geometric parameter. Note that when $d$ is very large the turbulence dissipation, Eq. (4), becomes very small. On the other hand, the strong tip vortices in the lower wake can generate significant turbulence production from Eq. (3). Over time, this imbalance in turbulence production and dissipation in the lower wake can result in excessively large eddy viscosities. These large viscosities can migrate up the vortex wake after several rotor revolutions, and under BVI conditions, infiltrate the blade boundary layers. When this happens, the rotor blade drag and torque increase significantly and artificially, resulting in large FM errors and an under-prediction of rotor efficiency.

The DES model is a RANS/LES hybrid approach that mitigates the problem of artificially large eddy viscosity. The turbulence length scale used in Eqns. 1-5 is modified by Eq. (6) by taking the minimum of the distance from the wall, $d$, and the local grid spacing, $\Delta=\max (\Delta \mathrm{x}, \Delta \mathrm{y}, \Delta \mathrm{z})$. The fixed coefficient is given by $C_{D E S}=0.65$.

$$
\bar{d}=\min \left(d, C_{D E S} \Delta\right)
$$

This simple but crucial change can be viewed in two different ways. From a numerical perspective the length scale in Eq. (6) has been significantly reduced which allows the turbulence dissipation in Eq. (4) to remain active in the vortex wake below the rotor plane. This prevents the turbulent eddy viscosity from growing to unrealistic values. The torque therefore remains unaffected, compared to the process described above, and the FM is accurately predicted. A physical interpretation views the modified length scale as an implicit filter, where the largest turbulent eddies are now grid-resolved on the order of size $\Delta$. All smaller eddies are modeled by a reduced turbulent eddy viscosity. This DES approach provides a rational way to reduce the length scale, and hence the turbulent eddy viscosity, based on a physical model.

The DES approach assumes that the wall-parallel grid spacing, $\Delta$, exceeds the thickness of the boundary layer 
so that the RANS model remains active near solid surfaces. If $\Delta<\delta$, the boundary layer thickness, then the DES Reynolds stresses can become under-resolved within the boundary layer and may lead to non-physical results, including grid-induced separation. Using delayed detached eddy simulation ${ }^{11}$ (DDES), the RANS mode is prolonged and is fully active within the boundary layer. The wall-parallel grid spacing used in this study does not violate the hybrid-LES validity condition. Thus DES and DDES should give similar results.

Another engineering approach is to simply zero out the right-hand side of Eq. (2) in the vortex wake, below the rotating blades. This zonal approach allows the turbulent eddy viscosity that is generated in the NB rotor-blade grids to convect downward relatively unchanged along a streamline into the lower-wake OB grids, i.e., except for numerical diffusion. In this way, the turbulent eddy viscosity is controlled and never allowed to grow to very large values, which can artificially reduce the FM. This approach is referred to as the LOB approach ${ }^{5}$ in OVERFLOW because it does not account for turbulence production, dissipation or diffusion in the lower wake OB grids. A comparison of Q-criterion using the DES and LOB models for $10^{\circ}$ collective is shown in Fig. 2. The difference in predicted FM between two models is less than 0.001 (the LOB model predicts a slightly higher FM). While two models compare well overall, the LOB model seems to show more scales in the wake away from the body and a wider spreading of the wake. More studies are needed to investigate the differences in wake structures.

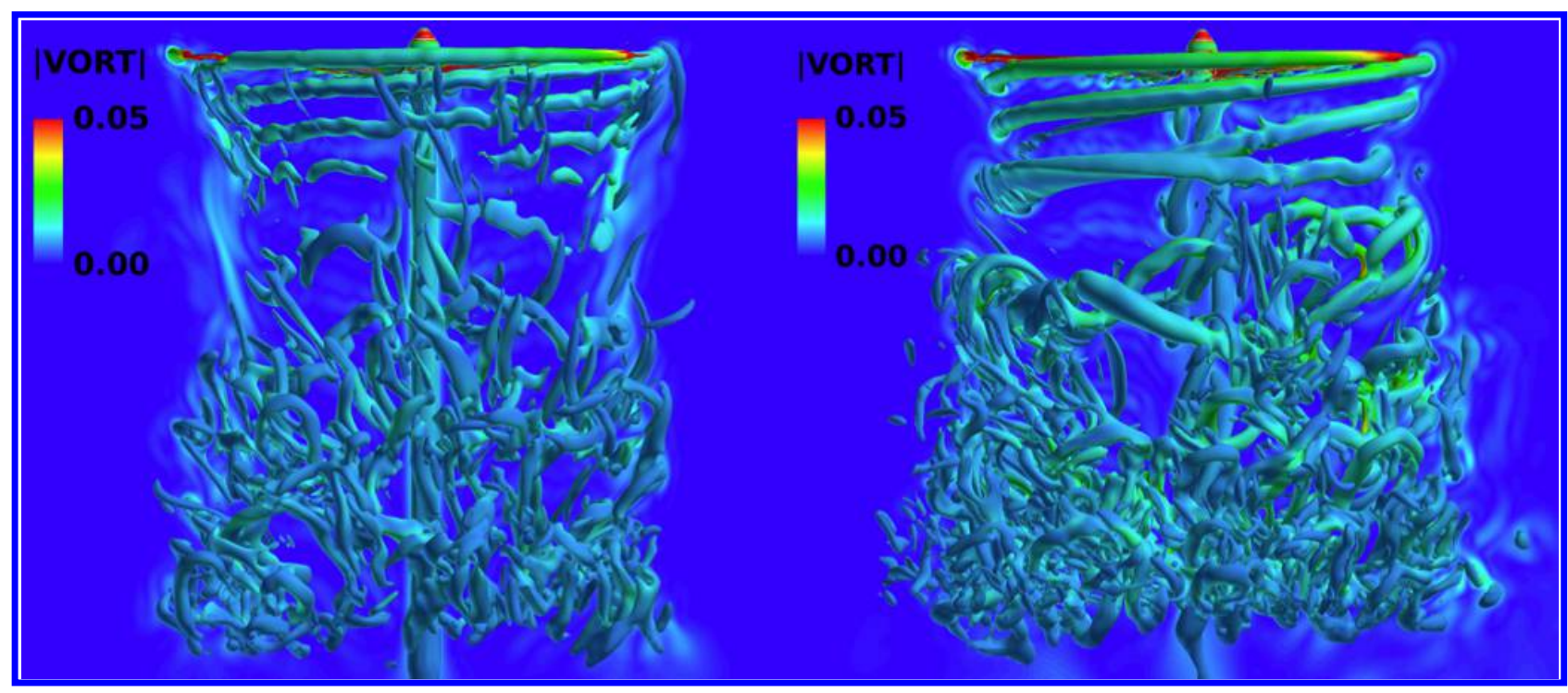

a) RANS(NB)-DES(OB)

b) RANS(NB)-LOB(OB)

Figure 2. Comparison of DES and LOB models for XV-15 rotor in hover. $\mathrm{M}_{\mathrm{tip}}=0.69, \operatorname{Re}_{\mathrm{Ctip}}=4.9$ million, $\theta=10^{\circ}$

\section{Results}

Three-dimensional unsteady fully turbulent flow simulations for an XV-15 rotor in hover were performed using OVERFLOW. The number of grid points used in this study was approximately 47 million. Grid resolution details are summarized in Table 1. Most results were produced using 2048 cores on the Pleiades system, a supercomputer that currently has 211,360 cores (162 racks) and a total of 723 TB memory at the NASA Advanced Supercomputing Division. The speed of each processor of 24-core Intel Xeon Haswell node is $2.5 \mathrm{GHz}$, and the available memory is 128 GB per node. A typical job requires approximately 24 hours of wall-clock time for 44,640 steps. When the size of the time step is $0.25 \mathrm{deg}, 1,440$ steps are needed for a single revolution. Since the first 1,440 steps uses a time step of $2.5 \mathrm{deg}$, the total number of steps is equivalent to 40 physical revolutions.

Figure 3 shows that the RANS model underpredicts FM for all collective angles for an isolated XV-15 rotor in hover. The experimental values of FM are from Ref. 12. The largest difference occurs at the $\theta=14$ deg collective (pitch) case. The local tip Mach number is 0.69 and the Reynolds number based on the tip chord is 4.9 million. Figure 4 shows the iso-surfaces of Q-criterion and TEV on a constant coordinate plane to compare the RANS(NB)- 
RANS(OB) and RANS(NB)-LOB(OB) solutions. The plotting range for TEV is $0-70,000$. The decreased turbulent dissipation in the wake of the RANS(OB) model does not balance the production of TEV. Figure 5 shows the Q-criterion and TEV on a different spectrum range of 0-300 to contrast the high TEV level on the iso-surfaces of the RANS $(\mathrm{OB})$ model to the low level of the $\mathrm{LOB}(\mathrm{OB})$ solution. Note: the red color means TEV $\geq 300$.

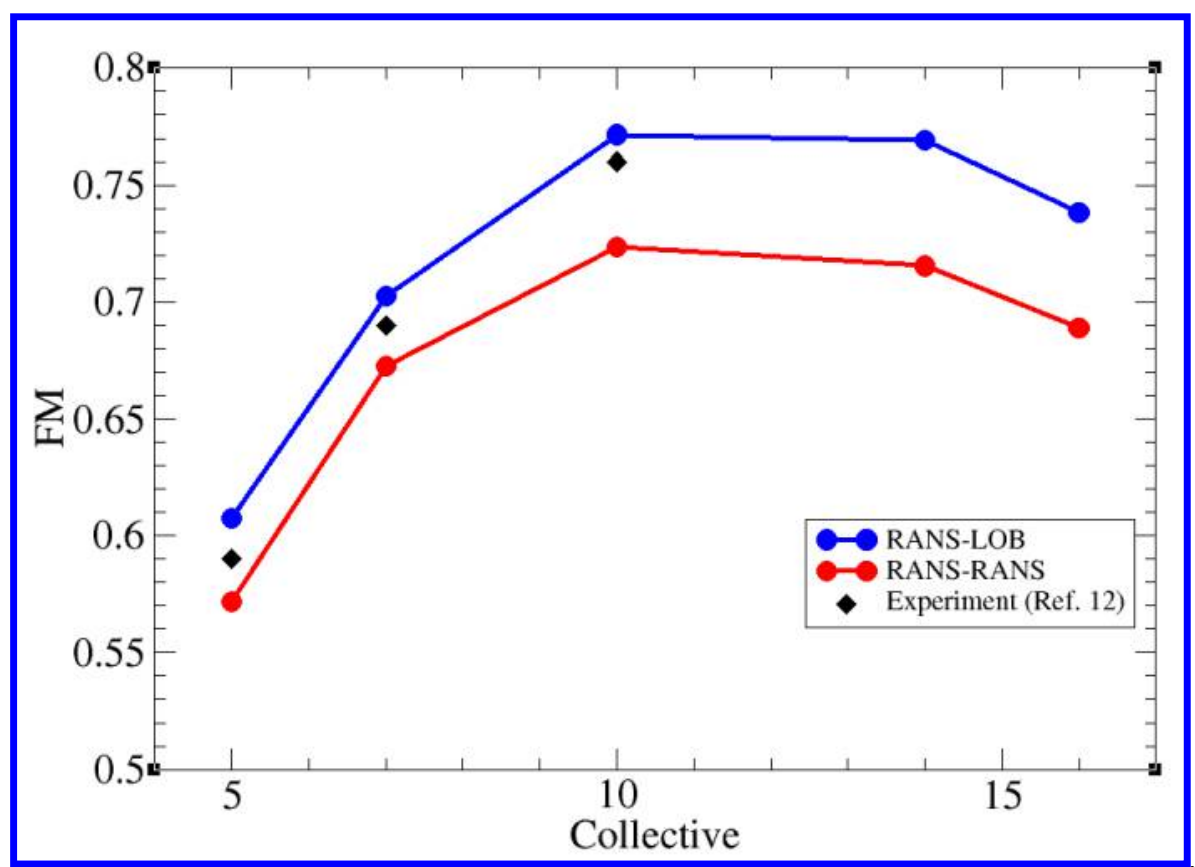

Figure 3. FM vs. Collective - Comparison of RANS and LOB models for off-body grids. $\mathrm{M}_{\text {tip }}=0.69, \operatorname{Re}_{\text {Ctip }}=4.9$ million

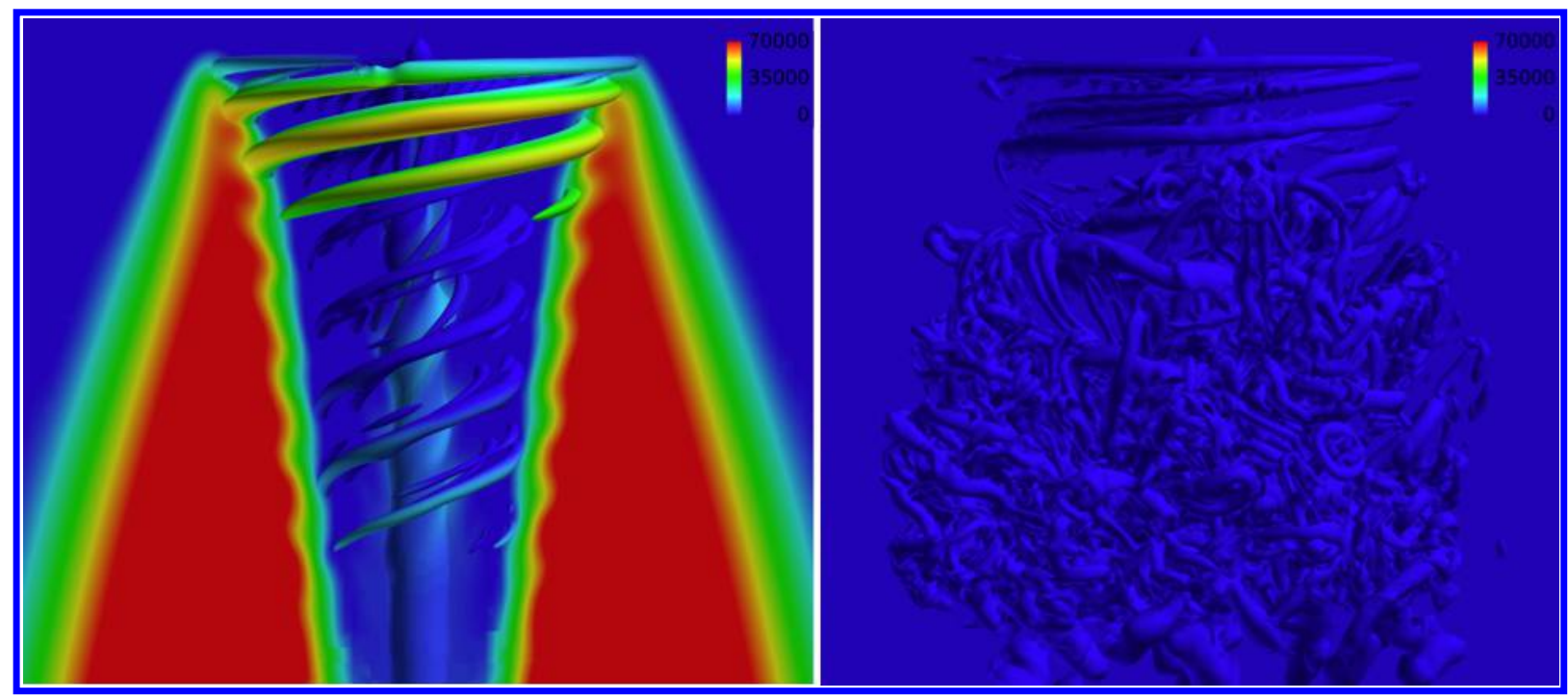

a) RANS(NB)-RANS(OB)

b) RANS(NB)-LOB(OB)

Figure 4. Q-criterion and TEV for XV-15 rotor in hover. $\mathrm{M}_{\text {tip }}=0.69, \mathrm{Re}_{\text {Ctip }}=4.9$ million, $\theta=14^{\circ}$, TEV range: $0-70,000$, where blue is low and red high on a constant coordinate plane. 


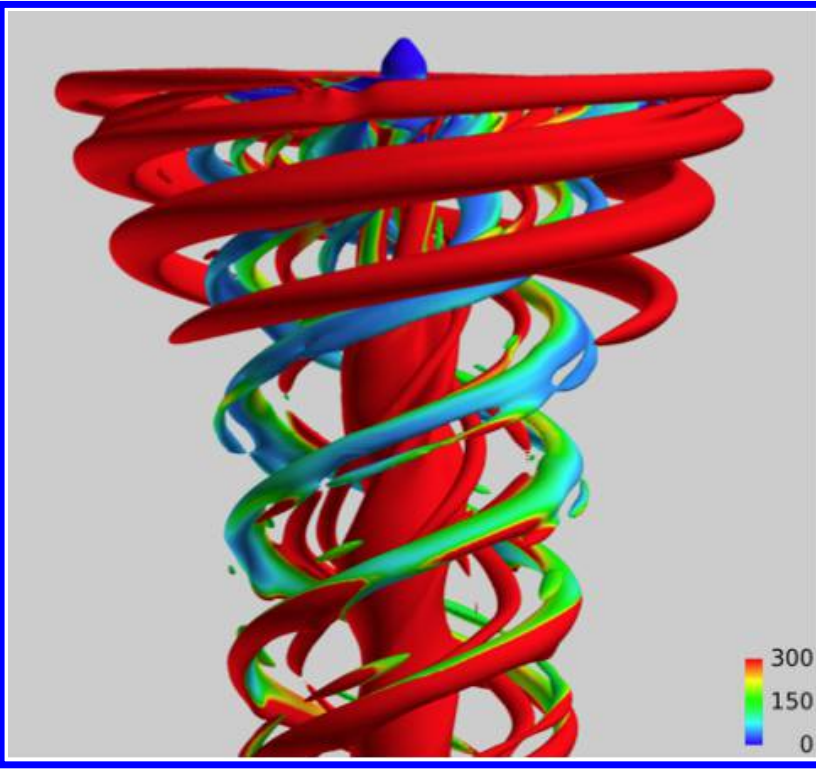

a) RANS(NB)-RANS(OB)

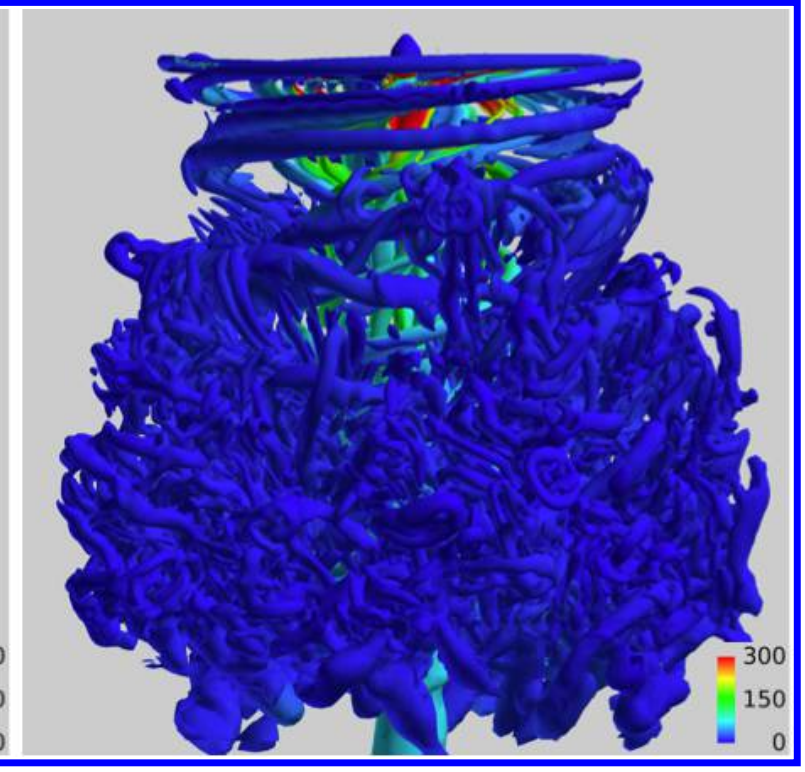

b) RANS(NB)-LOB(OB)

Figure 5. Q-criterion and TEV of $X V-15$ rotor in hover. $\mathrm{M}_{\text {tip }}=0.69, \mathrm{Re}_{\mathrm{Ctip}}=4.9$ million, $\theta=14^{\circ}$, TEV range: $0-300$ on iso-surfaces.

Figure 6 shows the TEV of the RANS(NB)-RANS(OB) model in the NB grids at various radial stations. The color spectrum range here is $0-10,000$. While the TEV at the inboard stations remains relatively low, it appears to be high at the outboard stations. Note that the high TEV advects from the lower side of the blade to the upper side at the most outboard station. Although the same model is used in the NB grids for both RANS(OB) and LOB(OB) solutions, the high TEV in the OB grids of the RANS $(\mathrm{OB})$ model convects into the NB grids. Figure 7 shows the velocity vectors colored by TEV at a mid-span station. The directions of velocity vectors suggest the downward induced flows near the leading edge at the inboard of the rotor. Figure 8 shows the velocity vectors at an outboard station. The flows near the leading edge of the rotor tip move upward indicating the advection of TEV. The velocity vectors near the trailing edge of the rotor tip also shows the advection of TEV in Fig. 9. High TEV in the suction side of the blade seems to reduce the speed of flow and hence increases pressure. More studies are needed to investigate the correlation between TEV and pressure. It is interesting to see a comparison of pressure coefficients for RANS $(\mathrm{OB})$ and $\mathrm{LOB}(\mathrm{OB})$ models in Fig. 10. The pressure on the upper surface of the $\mathrm{LOB}(\mathrm{OB})$ model is clearly lower than that of the RANS $(\mathrm{OB})$ model. Figure 11 shows that the difference in pressure on the lower surface looks less dramatic. Higher pressure of the RANS(OB) model on the upper surface results in an increase in torque and a decrease in thrust. Thus the FM, that is a ratio of thrust over torque, is lower for the RANS(OB) model than for the $\mathrm{LOB}(\mathrm{OB})$ model. 


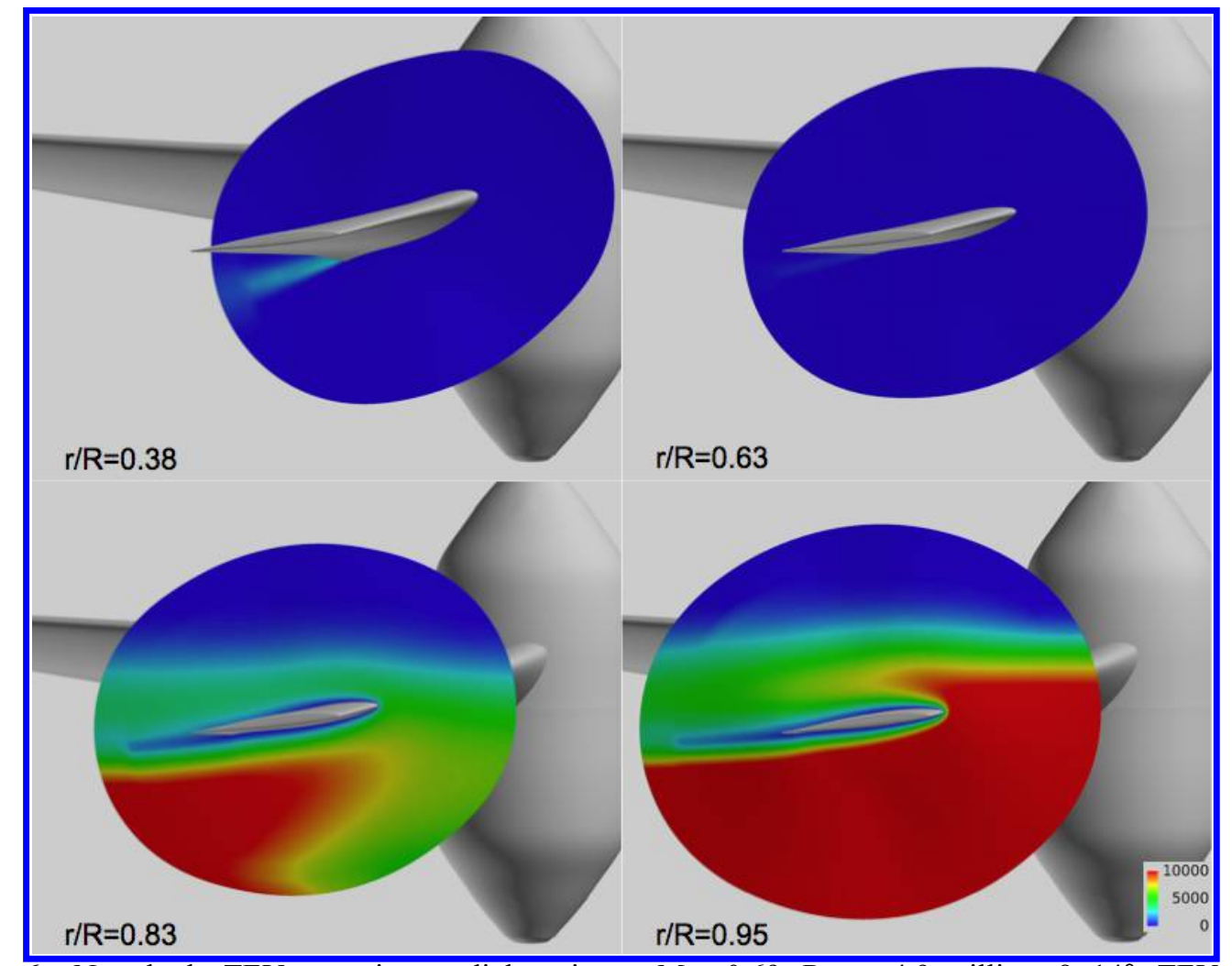

Figure 6. Near-body TEV at various radial stations. $\mathrm{M}_{\mathrm{tip}}=0.69, \mathrm{Re}_{\mathrm{Ctip}}=4.9$ million, $\theta=14^{\circ}$, TEV range: $0-10,000$, RANS(NB)-RANS(OB).

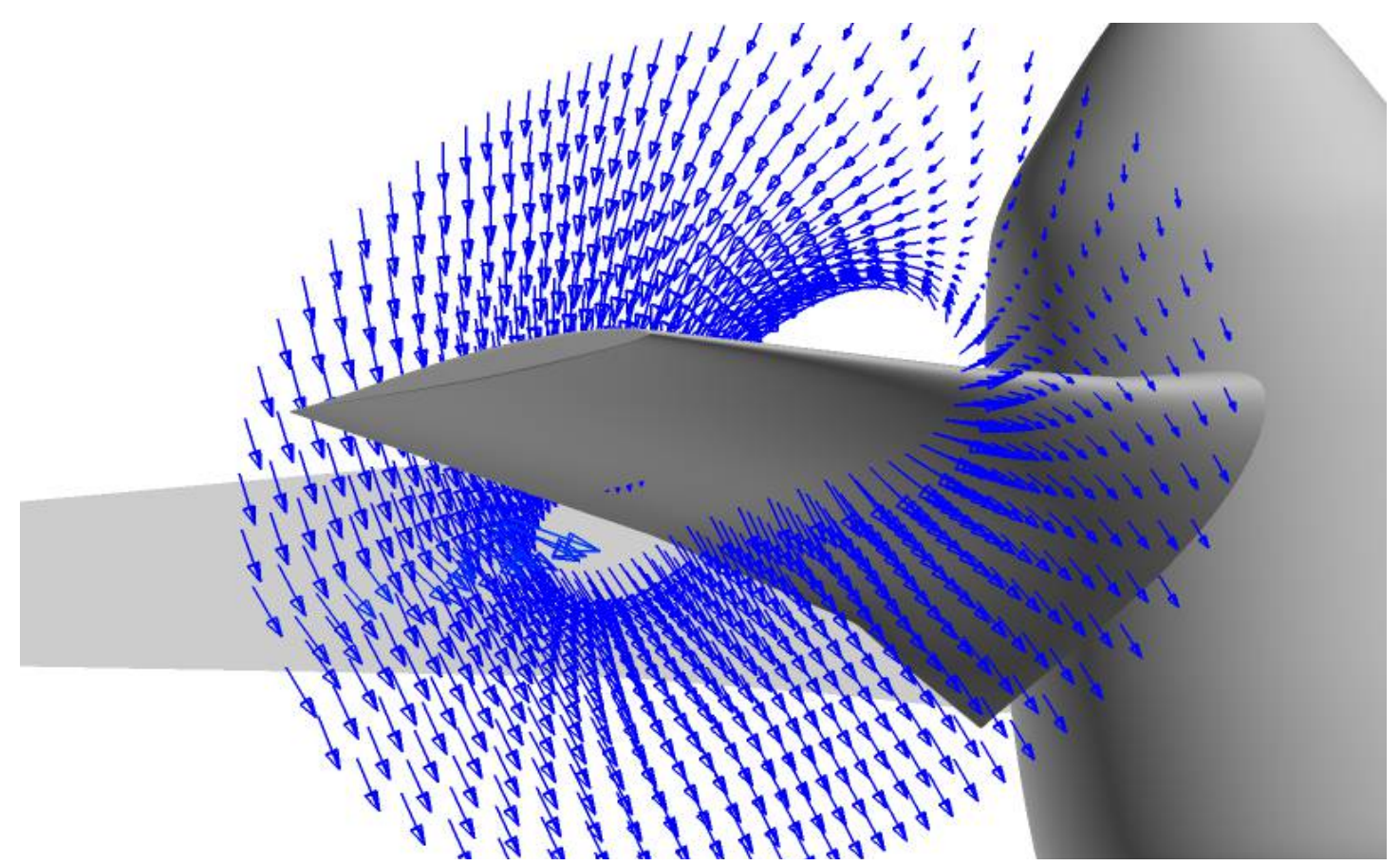

Figure 7. Near-body velocity vectors colored by TEV (red — high, blue — low) at $\mathrm{r} / \mathrm{R}=0.63$ radial station. $\mathrm{M}_{\mathrm{tip}}=0.69$, $\operatorname{Re}_{\text {Ctip }}=4.9$ million, $\theta=14^{\circ}$, RANS(NB)-RANS(OB). 


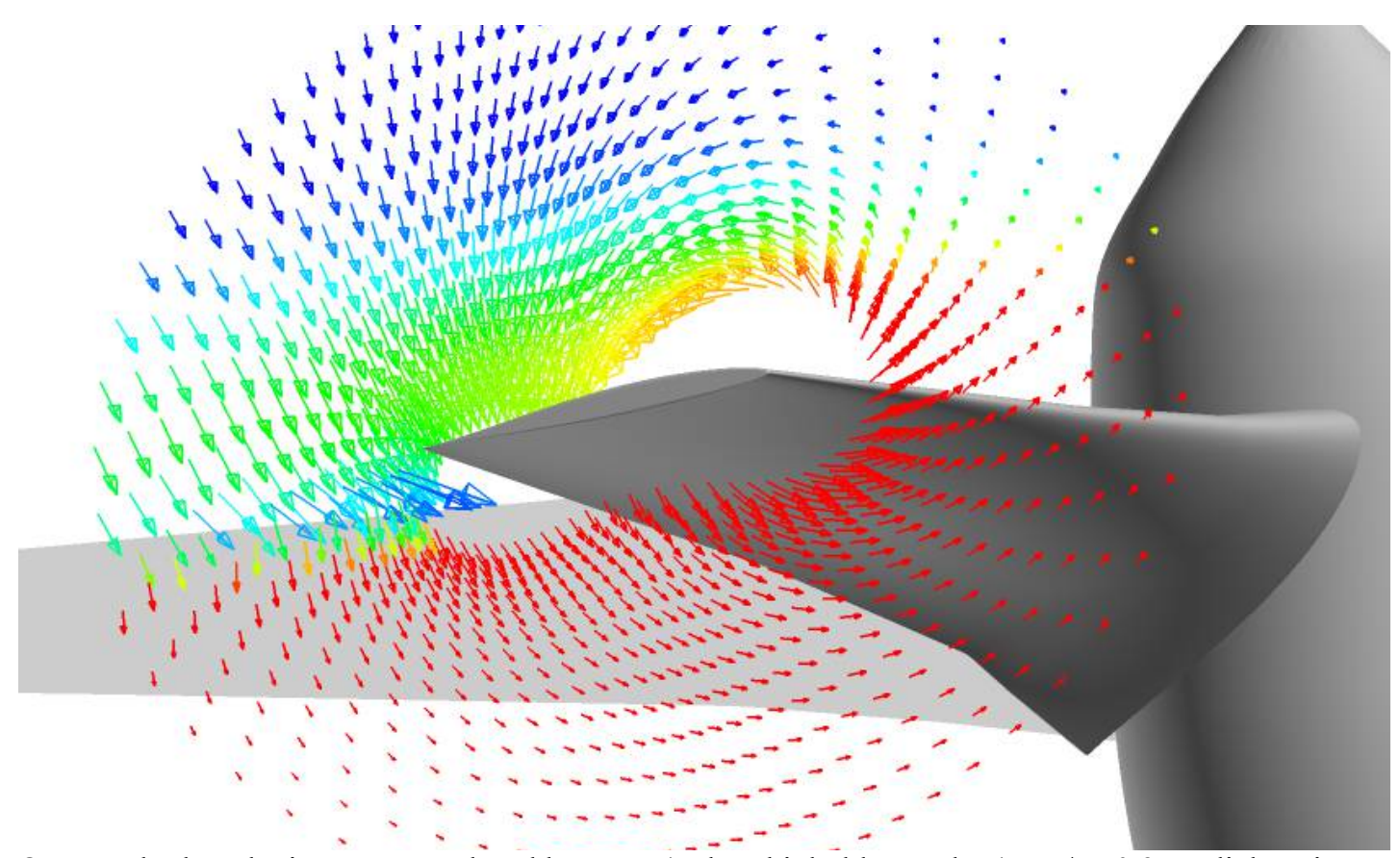

Figure 8. Near-body velocity vectors colored by TEV (red - high, blue - low) at $\mathrm{r} / \mathrm{R}=0.95$ radial station. $\mathrm{M}_{\text {tip }}=0.69$, $\operatorname{Re}_{\text {Ctip }}=4.9$ million, $\theta=14^{\circ}$, RANS(NB)-RANS(OB).

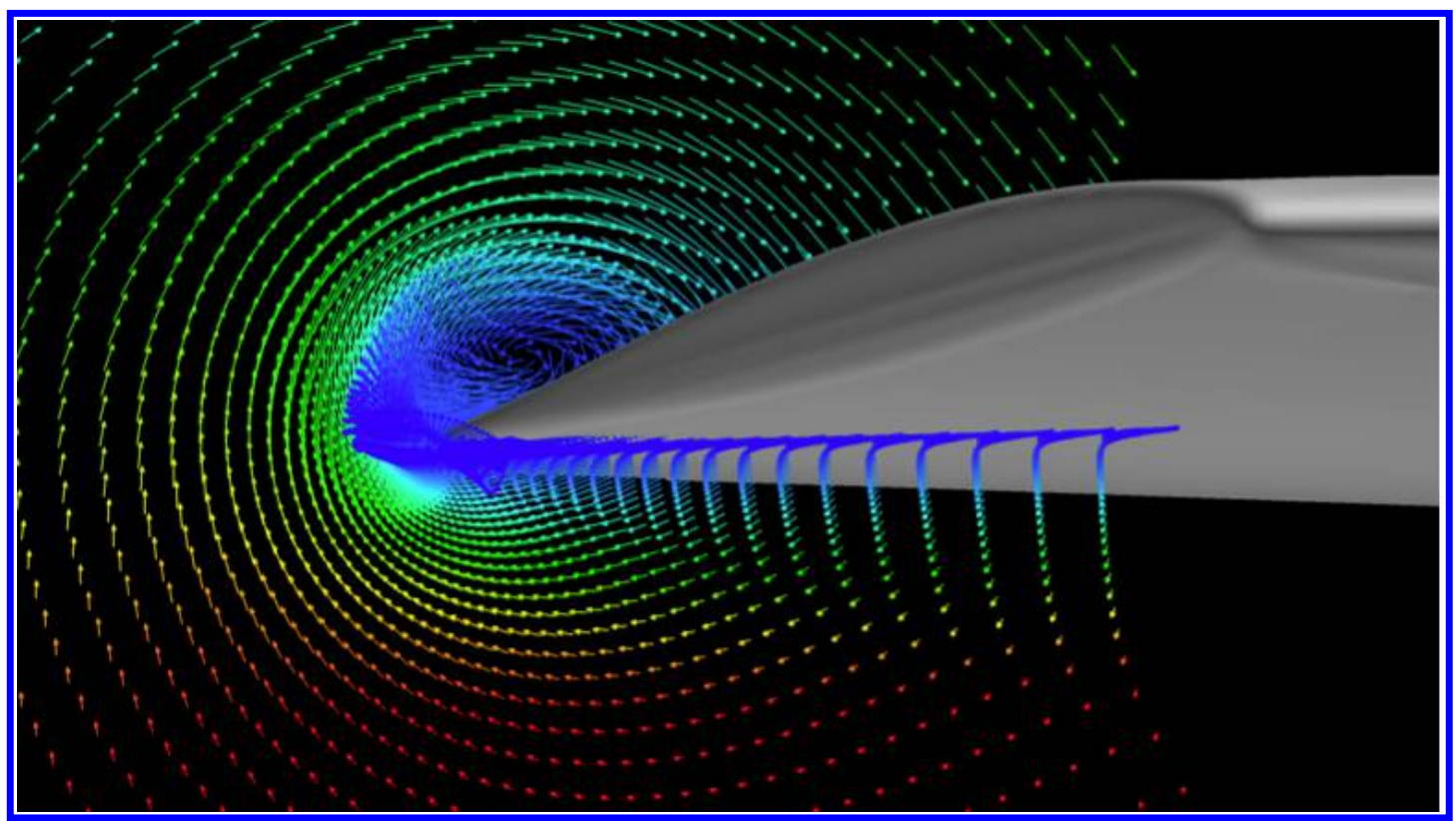

Figure 9. Near-body velocity vectors colored by TEV (red - high, blue - low) near the tip. $\mathrm{M}_{\text {tip }}=0.69, \operatorname{Re}_{\text {Ctip }}=4.9$ million, $\theta=14^{\circ}$, RANS(NB)-RANS(OB). 


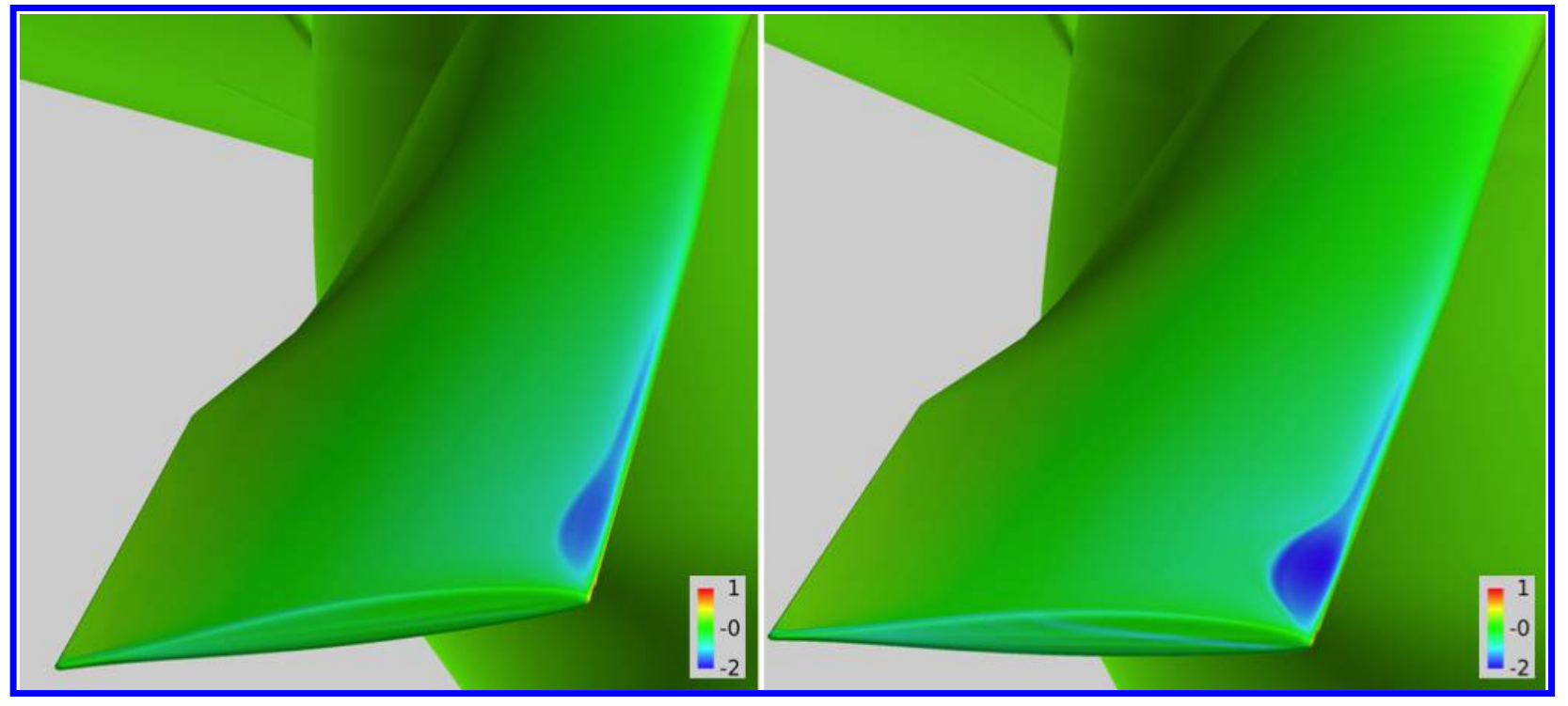

a) RANS(NB)-RANS(OB)

b) RANS(NB)-LOB(OB)

Figure 10. Upper surface $C_{p}$. $M_{\text {tip }}=0.69, R_{\text {Ctip }}=4.9$ million, $\theta=14^{\circ}, C_{p}$ range: $-2.0-1.33$.

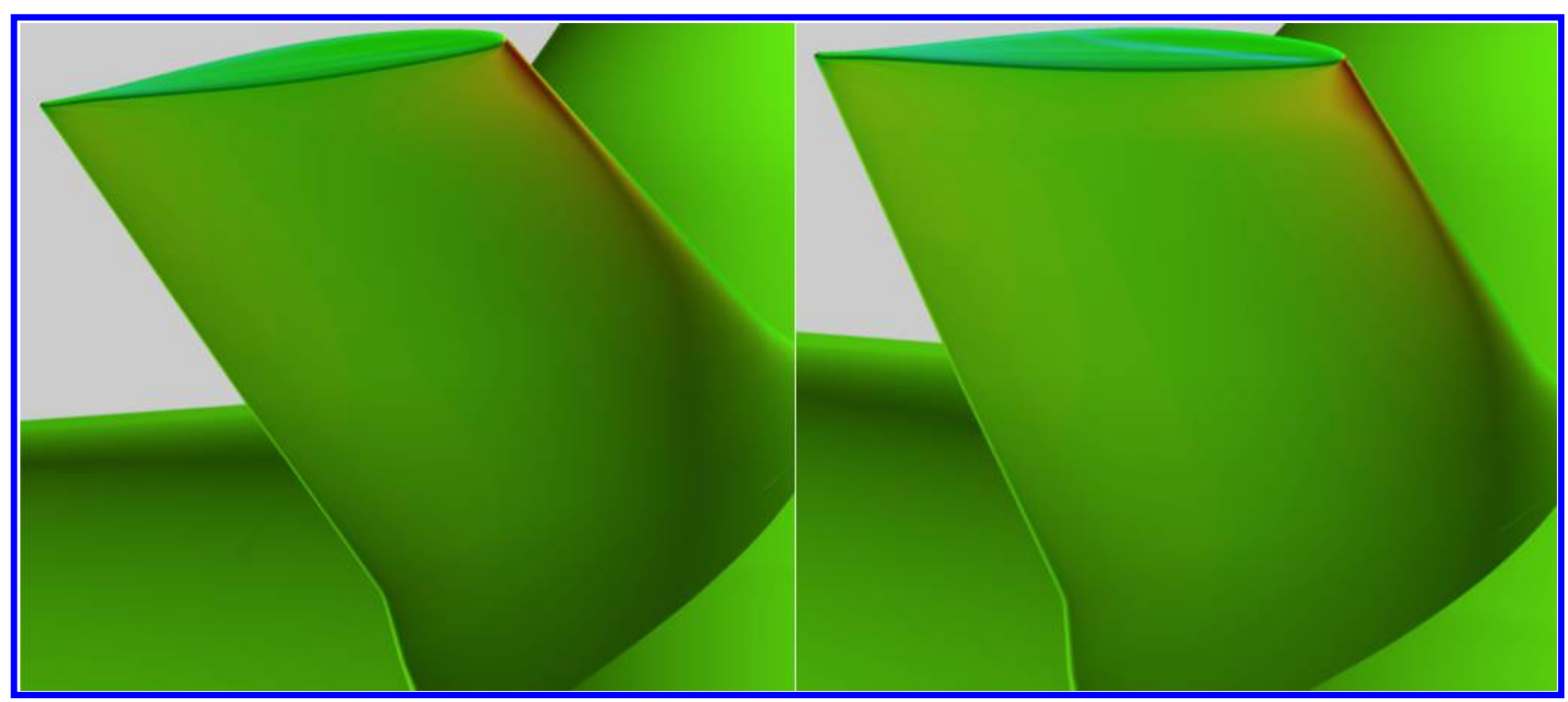

a) RANS(NB)-RANS(OB)

b) RANS(NB)-LOB(OB)

Figure 11. Lower surface $C_{p} . M_{\text {tip }}=0.69, \operatorname{Re}_{\text {Ctip }}=4.9$ million, $\theta=14^{\circ}, C_{p}$ range: $-2.0-1.33$. 
Table 2 compares the thrust, torque, and FM for the $\mathrm{LOB}(\mathrm{OB})$ and RANS $(\mathrm{OB})$ models. A two percent decrease in thrust and a four percent increase in torque resulted in a reduction of seven percent in FM for the RANS(OB) model.

\begin{tabular}{|c|c|c|c|c|c|c|}
\hline Model & Thrust (Ct) & Torque (Cq) & FM & \%Ct diff. & \%Cq diff. & \%FM diff. \\
\hline $\begin{array}{c}\text { RANS(NB)- } \\
\text { LOB(OB) }\end{array}$ & 0.01330 & 0.001410 & 0.769 & & & \\
\hline $\begin{array}{c}\text { RANS(NB)- } \\
\text { RANS(OB) }\end{array}$ & 0.01303 & 0.001471 & 0.715 & $-2.03 \%$ & $+4.33 \%$ & $-7.02 \%$ \\
\hline
\end{tabular}

Table 2. Effect of turbulence model on FM. $\mathrm{M}_{\mathrm{tip}}=0.69$, $\mathrm{Re}_{\mathrm{Ctip}}=4.9$ million, $\theta=14^{\circ}$.

Although the first idea of coaxial rotors appeared more than 150 years ago, most helicopters have used single main rotor/ tail rotor combination. However, recent interest in unmanned aircraft systems and high-speed rotorcraft has renewed interest in the coaxial configuration. Figure 12 shows a comparison of TEV for coaxial rotors with the separation distance of 0.25 rotor diameter. The collective is set to $10^{\circ}$ for both upper and lower rotors in this case for simplicity. While high TEV and subsequent dissipation of wake vortices for the lower rotor is anticipated, the RANS OB model captures the wake system of the upper rotor relatively well except for the vortex entering the plane of the lower rotor. An investigation of wall distance function in Fig. 13 shows that the values of the wall distance function appear to be small between the upper and lower rotors because the function is the distance to any nearest wall. Since the length scale of RANS model is the wall distance, the TEV between the upper and lower rotors remains relatively small.

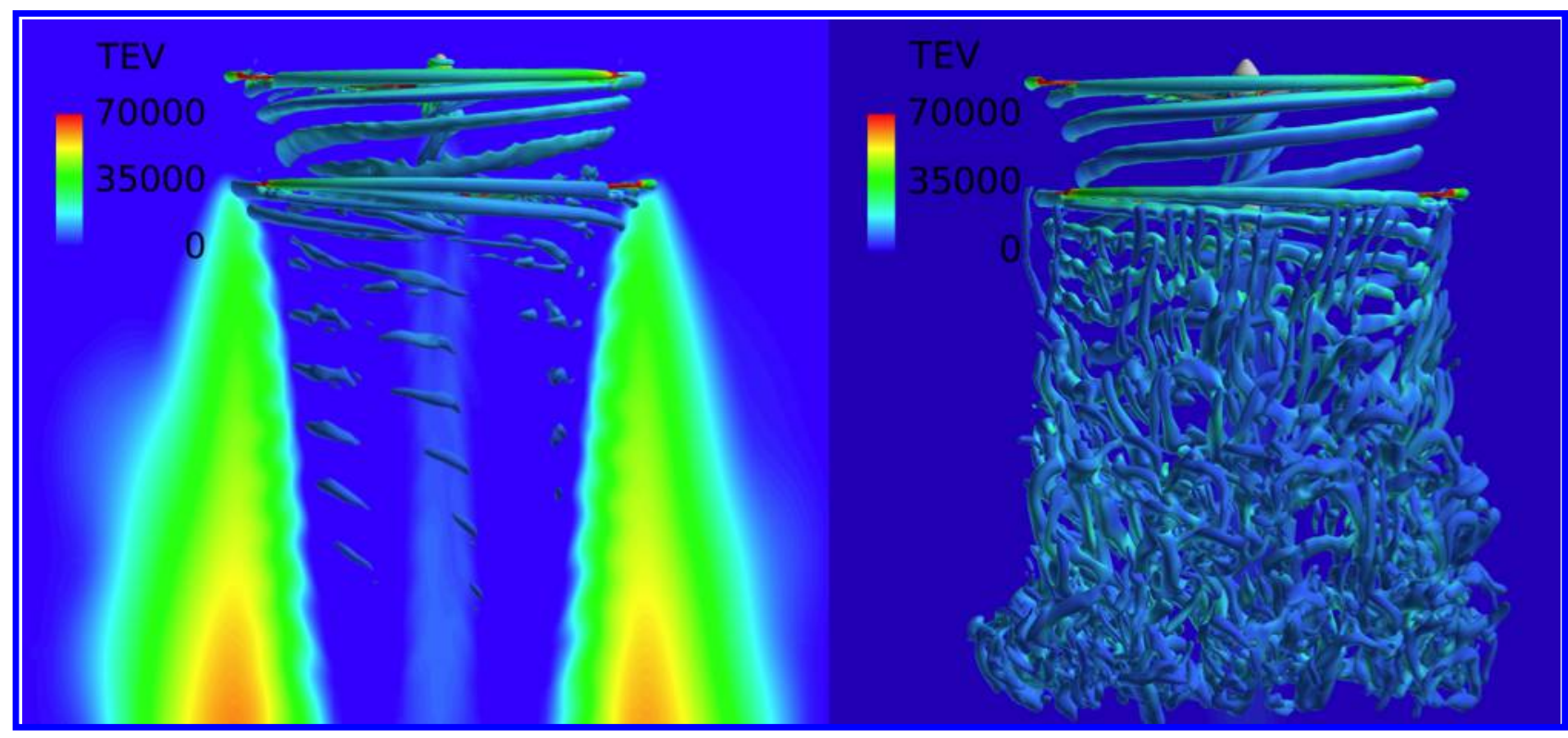

a) RANS(NB)-RANS(OB)

b) RANS(NB)-LOB(OB)

Figure 12. TEV for Coaxial Rotors, $\mathrm{M}_{\mathrm{tip}}=0.69, \mathrm{Re}_{\mathrm{Ctip}}=4.9$ million, $\theta=10^{\circ}$, TEV range: $0-70000$. 


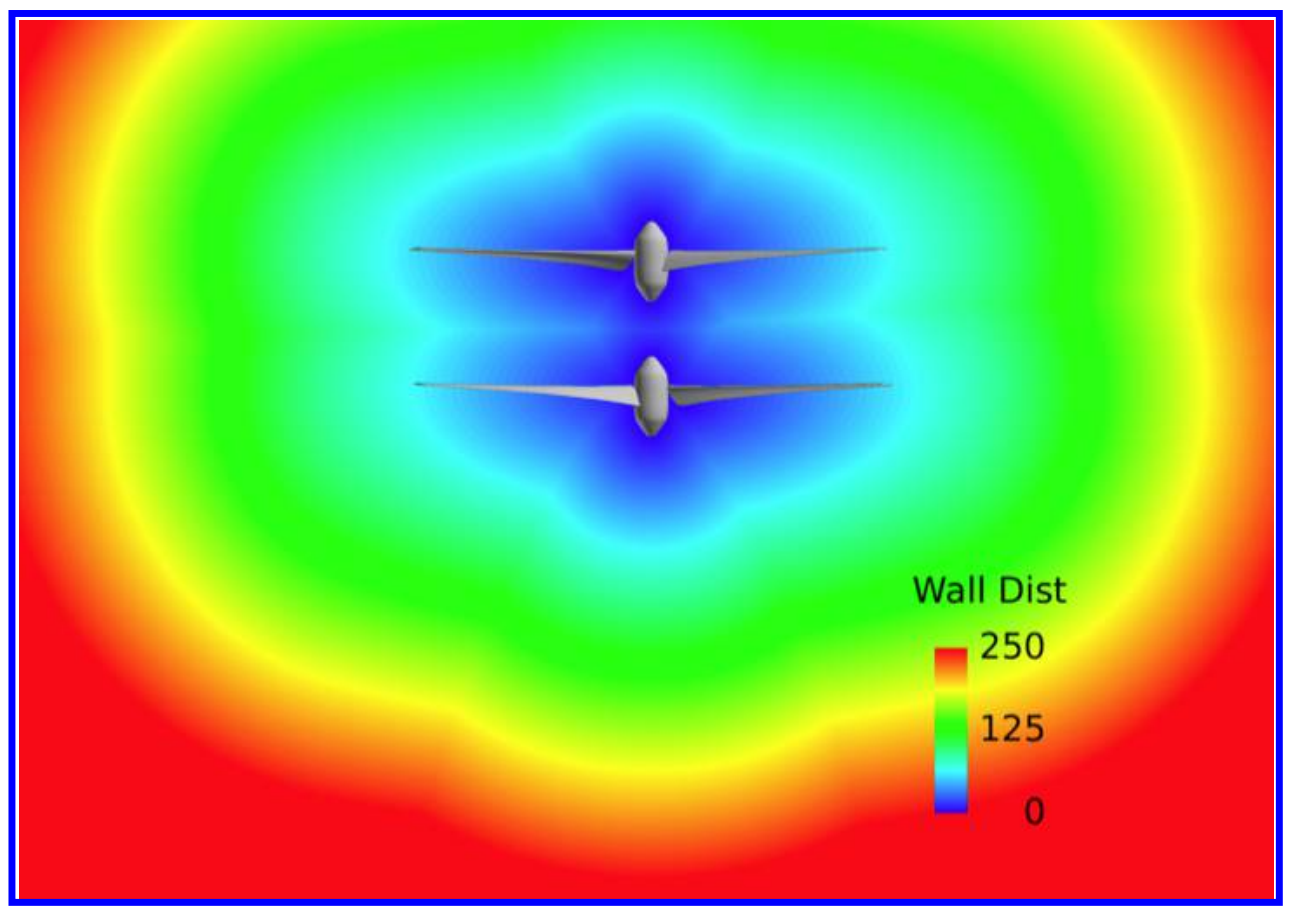

Figure 13. Wall distance function for coaxial rotors, $\mathrm{M}_{\mathrm{tip}}=0.69, \mathrm{Re}_{\mathrm{Ctip}}=4.9$ million, $\theta=10^{\circ}$.

\section{Conclusions}

A choice of turbulence models for off-body grids affects the accuracy of solutions generated by OVERFLOW code significantly. The LOB model compares favorably to the well-established DES for predicting FM for $\theta=10^{\circ}$. The LOB produces more vortical scales, consistent with the lower TEV, but the wake seems to spread wider than the DES. The RANS model underpredicts FM for all collectives compared to the LOB model. The directions of velocity vectors suggest the downward induced flows near the leading edge at the inboard of the rotor. However, at the outboard stations, the flows near the leading edge of the rotor move upward indicating the advection of TEV. High TEV for the RANS model on the suction side of the blade seems to reduce the speed of flow and hence increases pressure. More studies are needed to investigate the correlation between TEV and pressure. The pressure on the upper surface of the LOB model is lower than that of the RANS model. Higher pressure of the RANS model on the upper surface results in an increase in torque and a decrease in thrust. Thus, the FM, that is a ratio of thrust over torque, is lower for the RANS than for the LOB model. For coaxial rotors, the RANS model captures the wake vortices of the upper rotor well when the separation distance is relatively small because the values of wall distance function are small.

\section{References}

${ }^{1}$ Yoon, S., Pulliam, T. H., and Chaderjian, N. M., "Simulations of XV-15 Rotor Flows in Hover Using OVERFLOW," The $5^{\text {th }}$ Decennial AHS Aeromechanics Specialists' Conference, San Francisco, Jan. 2014.

${ }^{2}$ Chaderjian, N. M. and Ahmad, J. U., "Navier-Stokes Assessment of Test Facility Effects on Hover Performance," The AHS 71 ${ }^{\text {st }}$ Annual Forum, Virginia Beach, May 2015.

${ }^{3}$ Spalart, P. R., Jou, W-H., Strelets, M., and Allmaras, S. R., "Comments on the Feasibility of LES for Wings and on a Hybrid RANS/LES Approach," Advances in DNS/LES, Greyden Press, 1997, pp. 137-147.

${ }^{4}$ Chaderjian, N. M. and Buning, P., "High Resolution Navier-Stokes Simulation of Rotor Wakes," AHS $67^{\text {th }}$ Annual Forum, May 2011.

${ }^{5}$ Potsdam, M. and Pulliam, T. H., "Turbulence Modeling Treatment for Rotorcraft Wakes," AHS Aeromechanics Specialists Meeting, Jan. 2008.

${ }^{6}$ Spalart, P. R. and Allmaras, S. R., “A One-Equation Turbulence Model for Aerodynamic Flows,” AIAA Paper 1992-0439, Jan. 1992. 
${ }^{7}$ Nichols, R., Tramel, R., and Buning, P., "Solver and Turbulence Model Upgrades to OVERFLOW2 for Unsteady and High-Speed Flow Applications," AIAA Paper 2006-2824, June 2006.

${ }^{8}$ Pulliam, T. H., "High Order Accurate Finite-Difference Methods: as seen in OVERFLOW," AIAA Paper 20113851, June 2011.

${ }^{9}$ Holst, T. L. and Pulliam, T. H., "Overset Solution Adaptive Grid Approach Applied to Hovering Rotorcraft Flows," AIAA Paper 2009-3519, June 2009.

${ }^{10}$ Pulliam, T. H., and Chaussee, D. S., "A Diagonal Form of an Implicit Approximate-Factorization Algorithm," Journal of Computational Physics, Vol. 39, No. 2, 1981, pp. 347-363.

$\checkmark{ }^{11}$ Spalart, P. R., "Detached-Eddy Simulation," Annual Review of Fluid Mechanics, Vol. 41, 2009, pp. 181-202

${ }^{12}$ Betzina, M. D., "Rotor Performance of an Isolated Full-Scale XV-15 Tiltrotor in Helicopter Mode," AHS Technical Specialists Meeting, Jan. 2002. 